\title{
Influência do ciclo de polimerização sobre a dimensão vertical de oclusão em próteses totais
}

\author{
ANDREA LÚCIA ALMEIDA DE CARVALHO*; MARCO ANTONIO COMPAGNONI**; DÉBORA BARROS \\ $B A R B O S A^{* * *}$
}

\section{Resumo}

Alterações dimensionais na resina acrílica durante sua polimerização são bastante comuns, tendo como uma das implicações clínicas o aumento da dimensão vertical de oclusão em próteses totais. $\mathrm{O}$ objetivo do presente trabalho foi avaliar a influência de diferentes ciclos de polimerização em banho de água quente sobre a dimensão vertical de oclusão em próteses totais. Utilizou-se três ciclos para polimerização da prótese total superior, um ciclo longo de tempo, outro australiano e o último foi estabelecido empiricamente. Não existiu diferença estatisticamente significante entre os grupos avaliados e os resultados evidenciaram um aumento médio da dimensão vertical de oclusão inferior a $0,35 \mathrm{~mm}$ após a polimerização nos três ciclos propostos. No entanto, recomenda-se o ajuste oclusal para um correto restabelecimento da dimensão vertical de oclusão antes que as próteses totais sejam concluídas e entregues ao paciente.

\section{UNITERMOS}

Dimensão vertical; prótese total; resina acrílica; ciclo de polimerização.
CARVALHO A. L. A.; COMPAGNONI, M.A.; BARBOSA, D.B. The influence of polymerization cycle on occlusion vertical dimension of complete dentures. PGR-Pós-Grad Rev Fac Odontol São José dos Campos, v.4, n.3, set./dez. 2001.

\section{Abstract}

Dimensional changes in the acrylic resin base during polymerization procedure can cause increase in occlusion vertical dimension in complete dentures. The objective of this study was to evaluate the influence of different polimerization cycles in water bath on the occlusion vertical dimension in complete dentures. Three cycles were used to cure upper complete dentures, one for a long time, other similar to australian cycle and the last was established empirically. There was no significant difference between the groups and the results showed an average pin open to all groups evalueted under $0.35 \mathrm{~mm}$. However, the occlusal adjustment it is necessary to reestablish the occlusal vertical dimension before the complete dentures to be ended and given to the patient.

\section{UNITERMS}

Vertical dimension; complete denture; acrylic resin; polymerization cycle.

\footnotetext{
* Professora do Curso de Odontologia da Universidade Federal do Maranhão - 65020-240.

** Professor Titular da Disciplina de Prótese Total da Faculdade de Odontologia de Araraquara - UNESP - 14801-903.

*** Aluna do Programa de Pós Graduação em Reabilitação Oral - Nível Doutorado - Área de Prótese - Faculdade de Odontologia de Araraquara UNESP - 14801-903.
} 


\section{INTRODUÇÃO}

Muitas técnicas têm sido utilizadas para a polimerização do poli metil metacrilato (PMMA). Dentre essas técnicas temos a polimerização pelo calor em banho de água quente, a polimerização por luz visível, a polimerização química em temperatura ambiente e mais recentemente a polimerização ativada pela energia de microondas. A polimerização convencional em banho de água quente é ainda a mais utilizada, cerca de $95 \%,{ }^{14}$ onde a reação de polimerização é exotérmica e a quantidade de calor desprendida pode representar um fator importante na polimerização adequada da resina acrílica. Entretanto, para a obtenção de um método de polimerização efetivo vários fatores devem ser considerados, entre eles o volume da resina acrílica, a temperatura ambiente e a velocidade do aumento da temperatura durante a polimerização ${ }^{6}$. Em função disso, a polimerização em banho de água quente pode se dar através de ciclos longos ou curtos, executados em aparelho próprio que proporcione controle de tempo e temperatura. No entanto, na maioria das vezes, isso não acontece, ocorrendo a polimerização da resina acrílica de forma empírica, sem qualquer controle de tempo e/ou temperatura ${ }^{5}$, e segundo Anthony \& Peyton, ${ }^{1}$ existe uma correlação entre o grau de contração da resina acrílica e o calor aplicado durante o processo de polimerização.

Compagnoni et al. ${ }^{4}$ constataram alterações na oclusão e na DVO após a inclusão e polimerização de próteses totais, alterações estas que podem ser influenciadas pelos diferentes métodos de processamento da prótese ${ }^{10}$, entre eles a forma e o tipo de material utilizado para inclusão ${ }^{18}$, a prensagem da prótese $^{16}$ e o ciclo de polimerização utilizado ${ }^{13}$.
Considerando que a DVO, determinada clinicamente durante a confecção de prótese totais, necessita ser mantida após o seu processamento laboratorial, propôs-se avaliar neste trabalho as possíveis alterações verticais nestas próteses em função de três diferentes ciclos de polimerização da resina acrílica em banho de água quente.

\section{Material e Método}

Foram utilizadas trinta próteses totais superiores que foram divididas em três grupos de dez, de acordo com o ciclos de polimerização a serem avaliados, descritos no Quadro 1.

Uma prótese total superior e uma prótese total inferior foram confeccionadas a partir de um modelo padrão obtido de uma matriz de silicona, seguindo-se os conceitos laboratoriais preconizados por Russi et al. ${ }^{15}$, na seqüência abaixo descrita:

- confecção de bases de prova sobre um modelo superior e inferior padrão, utilizados para o ensino de graduação e Pós-Graduação na Disciplina de Prótese Total da Faculdade de Odontologia de Araraquara - UNESP;

- montagem arbitrária no articulador semiajustável (Cosmax, Type M.Y.-3, J. Morita Corporation) e montagem dos dentes artificiais 263/2N (Dentsply Brasil, Ind. Com. Ltda).

Optou-se pela presença de dentes artificiais para aproximar esse trabalho experimental das condições realizadas na prática clínica, pois, segundo Baemmert et al. ${ }^{3}$, a ausência de dentes artificiais pode interferir na estabilidade dimensional das bases de resina acrílica de próteses totais.

\section{QUADRO 1 - Descrição dos ciclos de polimerização em banho de água quente.}

A Imersão da mufla em água em temperatura ambiente, elevando-se a temperatura para $74^{\circ} \mathrm{C}$ e permanecendo nessa temperatura por 9 horas ${ }^{13}$

B Aquecimento da mufla da temperatura ambiente até $65^{\circ} \mathrm{C}$ por 30 minutos, permanecendo nesta temperatura por 1 hora. A seguir elevou-se a temperatura até $100^{\circ} \mathrm{C}$ durante 30 minutos e esta temperatura foi mantida por mais uma hora ${ }^{17}$

C Aquecimento da mufla em fogo alto no interior de um caldeirão contendo água em temperatura ambiente e, manter por 1 hora em fogo baixo a partir do momento em que a água entrar em ebulição, resultando num total aproximado de 2 horas ${ }^{5}$ 
Procedeu-se inicialmente a inclusão, prensagem e polimerização da prótese total inferior de maneira convencional.

Após a polimerização e desinclusão, a prótese total inferior foi remontada no articulador e estando a prótese total superior ainda em cera, realizouse o ajuste oclusal desgastando-se, quando necessário, somente os dentes artificiais da prótese total inferior.

Após esses procedimentos, o conjunto modelo superior/prótese total superior encerada foi removido do articulador e um molde deste conjunto foi obtido em silicona industrial (RTV-3120, Reforplás Ind. e Com. Ltda), servindo assim, como uma matriz para a obtenção dos corpos-de-prova, Figura 1.

No interior dessa matriz os dentes artificias de resina acrílica (modelo 263/30M) foram adaptados em suas respectivas lojas, verteu-se no seu interior cera 7 liqüefeita e em seguida o modelo desdentado superior foi acomodado sobre a cera. Depois do resfriamento completo da cera, removeu-se o conjunto modelo superior/prótese total em cera, obtendo-se, dessa forma, o corpo-de-prova (Figura 2) padronizado quanto a espessura da base da prótese total superior e quanto ao posicionamento dos dentes artificias em relação a prótese total inferior já acrilizada.

Para a avaliação das alterações na DVO duas mensurações foram realizadas. A primeira, após a montagem do corpo-de-prova ainda em cera no articulador, tendo-se o cuidado de observar o toque do pino incisal sobre a mesa incisal, e a segunda mensuração foi realizada após a polimerização e remontagem do corpo-de-prova. Essas mensurações deram-se através de um relógio comparador (Baker-JO8) com precisão de $0,01 \mathrm{~mm}$, o qual era apoiado em um ponto fixo sobre o ramo superior do articulador que era posicionado sobre uma mesa de desempeno (Figura 3).

Dessa forma, na primeira mensuração o pino incisal sempre tocava a mesa incisal do articulador, enquanto que, após a acrilização e remontagem do corpo-de-prova, este pino poderia estar afastado da mesa incisal de forma que o ramo superior do articulador realizaria uma pressão sobre o sensor do relógio, registrando o valor vertical da segunda mensuração. Caso o pino incisal tocasse a mesa incisal após a acrilização e remontagem do corpo-de-prova, o mesmo era removido para se verificar se a coincidência era proveniente de uma ausência de alteração (corpo-de-prova 9 do ciclo C) ou se ocorreu uma alteração negativa (corpos-de-provas 8 e 10 do ciclo C). Para cada corpo-de-prova e nas duas etapas das mensurações, três medidas verticais foram tomadas registrando-se somente a média. A diferença da média entre a segunda mensuração e a primeira, quando existente, correspondeu a alteração da DVO nas próteses totais.

Cada corpo-de-prova foi polimerizado individualmente e sob prensa nos três ciclos em banho de água propostos anteriormente. Para o ciclo A, preconizado por Phillips ${ }^{13}$, e para o ciclo B, ciclo australiano ${ }^{16}$, utilizou-se uma polimerizadora automática (Termotron P-100, Termotron Equipamentos) com controle de tempo e temperatura. Já para o ciclo $\mathrm{C}$, ciclo empírico ${ }^{5}$ o aquecimento da água deu-se no interior de uma panela através de um fogão a gás convencional. Nesse ciclo não ocorreu o controle de temperatura, apenas foi observado o tempo de polimerização de 2 horas.

Todo o cuidado foi dado aos corpos-de-prova quando de sua inclusão realizando-se o isolamento do modelo préviamente a mesma, com vaselina sólida, para que fosse facilitado a sua desinclusão após a acrilização sem que nenhum dano ocorresse no mesmo que viesse a interferir na sua remontagem, a qual foi realizada seguindo os critérios preconizados por Lauritzen ${ }^{7}$ A preservação do modelo foi fator determinante no trabalho, e caso ocorresse alguma alteração no mesmo, um novo corpo-de-prova seria feito em substituição a esse. Deve-se salientar que cada corpo-de-prova foi montado individualmente no articulador em relação a prótese total inferior que foi única para todo experimento realizado. 


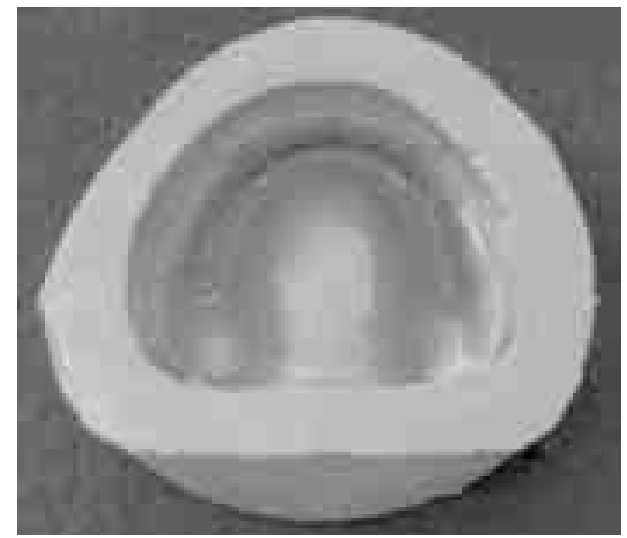

FIGURA 1 - Matriz em silicona para obtenção dos corpos-de-prova.

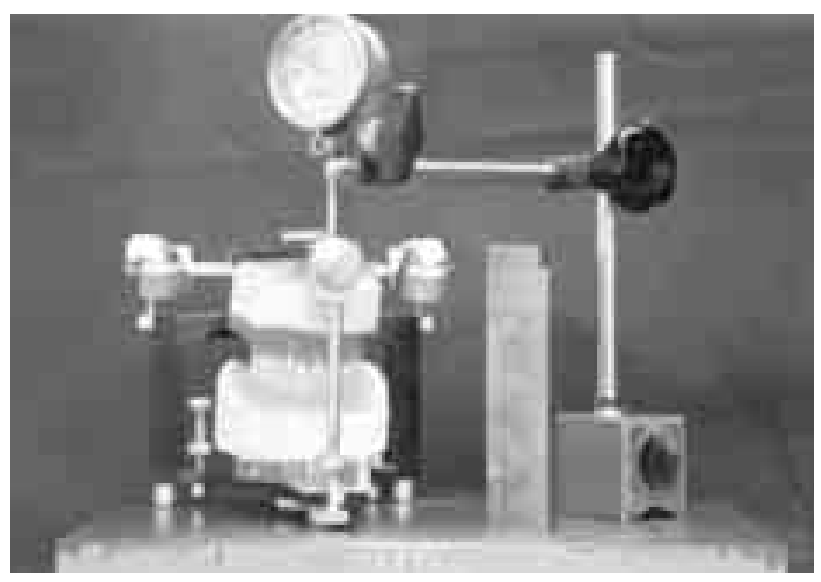

\section{Resultados}

Para os ciclos A e B, ocorreram aumentos na DVO após a polimerização da prótese total superior. No entanto, para o ciclo C, dois corpos-de-prova após a polimerização tiveram a DVO reduzida. A média das alterações verticais para os ciclos A, B e $\mathrm{C}$ foram respectivamente de $0,335 \mathrm{~mm}, 0,236 \mathrm{~mm}$ e $0,233 \mathrm{~mm}$, como pode ser evidenciado na Tabela 1 .

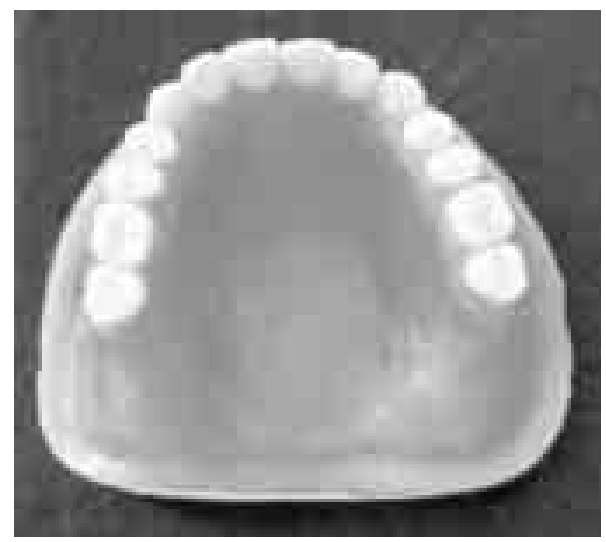

FIGURA 2 - Corpo-de-prova em cera.

FIGURA 3 - A) articulador; B) relógio comparador; C) base de suporte; D): mesa de desempeno.

A Tabela 2 mostra que o valor de F correspondente ao fator de variação (ciclo de polimerização) não foi significante, pois $p$ foi maior que 0,05 . Assim, houve evidência amostral para aceitar-se a hipótese de que o ciclo de polimerização induziu efeitos semelhantes sobre a DVO das próteses totais. Não houve, portanto, diferença estatisticamente significante entre os três ciclos de polimerização avaliados em relação à alteração da DVO das próteses totais. 
Tabela 1 - Valores das alterações na dimensão vertical de oclusão, de acordo com os ciclos de polimerização (milímetros).

\begin{tabular}{|c|c|c|c|}
\hline & CICLO A & CICLO B & CICLO C \\
\hline & 0,56 & 0,14 & 0,36 \\
\hline & 0,47 & 0,12 & 0,19 \\
\hline & 0,44 & 0,24 & 0,30 \\
\hline & 0,59 & 0,24 & 0,54 \\
\hline & 0,05 & 0,16 & 0,62 \\
\hline & 0,17 & 0,11 & 0,28 \\
\hline & 0,18 & 0,07 & 0,24 \\
\hline & 0,24 & 0,22 & $-0,10$ \\
\hline & 0,05 & 0,40 & 0,00 \\
\hline & 0,60 & 0,66 & $-0,10$ \\
\hline Média & 0,335 & 0,236 & 0,233 \\
\hline$d p$ & 0,22 & 0,18 & 0,25 \\
\hline
\end{tabular}

Tabela 2 - Análise de variância da alteração da DVO.

\begin{tabular}{lcccc}
\hline Fonte de Variação & G.L. & S.Q. & Q.M. & F \\
Polimerização & 2 & 0,0674 & 0,0337 & 0,72 (n.s) \\
Resíduo & 27 & 1,2615 & 0,0467 & \\
\hline Total & 29 & 1,3289 & & \\
\hline
\end{tabular}

(n.s.) diferença não significava $-p>0,05$

\section{Dıscussão}

No presente trabalho observou-se que independente do ciclo de polimerização utilizado, ocorreram alterações na DVO das próteses totais. Badawy ${ }^{2}$ encontrou aumento médio de $0,75 \mathrm{~mm}$ após a polimerização de ambas as próteses totais, superior e inferior e, segundo Peyton et al. ${ }^{12}$ quando todos os cuidados são tomados durante o processamento da prótese, alterações verticais de até $0,50 \mathrm{~mm}$ são consideradas aceitáveis.

Observou-se, também, através de nossos resultados, que a alteração da DVO promovida pelos três ciclos avaliados foram semelhantes entre si, estan- do de acordo com os dados encontrados por Gonini et al. ${ }^{5}$ e Harman ${ }^{6}$ que não encontraram alterações dimensionais significantes na resina acrílica polimerizada em diferentes ciclos em banho de água quente. Phillips ${ }^{13}$ no entanto, relatou que vários fatores interferem na alteração dimensional da base de resina acrílica da prótese total, entre eles a contração de polimerização e a liberação de tensões da resina decorrentes de seu processamento laboratorial, provocando, com isso, modificações no posicionamento original dos dentes artificias e, consequentemente, alteração na DVO das próteses totais.

Embora em nosso trabalho não tenha ocorrido diferença estatisticamente significante entre os três 
ciclos, observou-se que o ciclo curto promoveu alteração vertical menor em relação ao ciclo longo e praticamente semelhante ao ciclo empírico avaliado. Já McCartney ${ }^{9}$ observou que as resinas acrílicas polimerizadas através do ciclo curto promoveram um aumento $50 \%$ maior na alteração da oclusão e 25\% maior na distorção da base da prótese na região média do palato, quando comparado ao ciclo longo. Com isso, esse último autor concluiu que ciclos de polimerização com baixas temperaturas que não utilizam ebulição no final do processo de polimerização promovem menos alterações na oclusão de próteses totais superiores.

As alterações dimensionais trazem como conseqüência a movimentação dos dentes artificiais, que, segundo Mahler ${ }^{8}$ são visualizadas pelo aumento da DVO. A esse respeito, esse mesmo autor comentou que um aumento de $1 \mathrm{~mm}$ da DVO pode dar-se como resultado da somatória do deslocamento individual de $0,01 \mathrm{~mm}$ de cada dente artificial da prótese total.

Acreditamos que as alterações oclusais e na DVO de próteses totais são consideradas clinicamente significantes, agravando-se principalmente em pacientes portadores de prótese total dupla, o que nos leva a indicar a remontagem destas próteses após sua acrilização, independente do ciclo de polimerização utilizado. Dessa forma, através desse procedimento, contatos prematuros oclusais se-

\section{RefERÊnCIAS Bibliográficas}

1. ANTHONY, D.H.; PEYTON, F. A. Dimensional accuracy of various denture base materials. J Prosthet Dent, v.12, p.67-81, 1962.

2. BADAWY, M. S. Occlusal vertical dimension changes in visible light-cured resin. Egypt Dent J, v.38, p.99-104, 1992.

3. BAEMMERT, R. J. et al. The effects of denture teeth on the dimensional accuracy of acrylic resin. Int J Prosthet, v.3, p. 528-37, 1990.

4. COMPAGNONI, M.A., et al. Alteração da dimensão vertical nas bases de dentaduras completas. Estudo comparativo entre duas técnicas de isolamento do gesso durante a inclusão. Rev Assoc Paul Cir Dent, v.37, p.49-54, 1983.

5. GONINI JUNIOR, A., et al. Influência dos ciclos na polimerização das RAAT: Influência de dois ciclos de polimerização das RAAT na exotermia da reação. Rev Assoc Paul Cir Dent, v.44, p.343-4, 1990. rão eliminados, evitando, com isso, que forças oclusais indesejáveis atuem sobre o rebordo alveolar e levem a sua reabsorção precocemente. Paralelamente serão precisos estudos adicionais relacionados ao ciclo de polimerização a ser aplicado visando observar qual a melhor maneira de se efetuar esse processo, considerando as outras propriedades físicas da resina acrílica.

\section{Conclusão}

Os resultados obtidos nesse trabalho permite-nos concluir que:

1. independente do ciclo de polimerização utilizado, houve alteração da DVO nas próteses totais após a polimerização da prótese total superior;

2. os ciclos A, B e C não mostraram diferenças estatisticamente significantes quanto as alterações verticais, prevalecendo aumentos médios de $0,335 \mathrm{~mm}, 0,236 \mathrm{~mm}$ e $0,233 \mathrm{~mm}$, respectivamente para cada um dos ciclos em banho de água quente avaliados.

\section{Agradecimentos}

À Fundação de Amparo à Pesquisa do Estado de São Paulo - FAPESP. Bolsa de Mestrado, processo $\mathrm{n}^{\circ}$ 96/03674-5.
6. HARMAN, I. M. Effects of time and temperature on polymerization of a methacylate resin denture base. J Am Dent Assoc, v.38, p.188-203, 1949.

7. LAURITZEN, A. Atlas de analisis oclusais. Madri: Ed. H.F. Martinez de Murguia, 1977. cap.11, p.181-6.

8. McCARTNEY, J. W. Flange adaptation discrepancy, palatal base distortion and induced malocclusion caused by processing acrylic resin maxillary complete dentures. J Prosthet Dent, v.52, p.545-53, 1984.

9. MAHLER, D.B. Inarticulation of complete dentures processed by compression molding technique. J Prosthet Dent, v.1, p. 5519, 1951.

10. NELSON, M. W.; KOTWAL, K. R.; SEVEDGE, S. R. Changes in vertical dimension of oclusion in conventional and microwave processing of complete dentures. J Prosthet Dent, v.65, p.306-8, 1994.

11. NOGUEIRA, S.S.; OGLE, R.E.; DAVIS, E.L. Comparision of accuracy between compression and injection-molded complete denture. J Prosthet Dent, v.82, p.291-300, 1999. 
12.PEYTON, F. A. et al. Materiales dentales restauradores. Buenos Aire: Mundi, 1960. p.383.

13.PHILLIPS, R.W. Materiais dentários de Skinner. 9.ed. Rio de Janeiro: Guanabara Koogan, 1993. 103-23p.

14. RUDD, K. D. Processsing complete dentures. Dent Clin North Am, v. 40, p.121-49, 1996.

15.RUSSI, S.; LOMBARDO, J.G.; COMPAGNONI, M.A. Prótese total manual de laboratório (teoria e prática). Araraquara: Faculdade de Odontologia, 1978, 91p.
16. RUSSI, S., et al. Dimensão vertical de bases de dentaduras completas. Influência de prensas para polimerização. Rev Gaucha Odontol, v.44, p.99-101, 1996.

17.TUCKFIELD, W.J.; WORNER, H.K.; GUERIN, B.D. Acrylic resin in dentistry, part. II. Aust Dent J, v.47, p.1-25, 1943.

18.ZAKHARI, K.N. Relationship of investing medium to occlusal changes and vertical opening during denture construction. J Prosthet Dent, v.36, p.501-9, 1976. 